\title{
Innovative Manufacturing and Joining Technologies for Aerospace Components
}

October saw the beginning of the EU project "Advanced Manufacturing Routes for Metal/Composite Components for Aerospace", or ADMACOM. The research teams involved want to find out how aircraft components manufactured using a combination of various materials can be economically produced. There must, of course, be no impairment in their functionality or safety.

T he advantages of modern materials are obvious: CFRPs are extremely strong, corrosion resistant and lightweight. And the combination of different materials of which the composites are made enhances the positive characteristics of each individual component. Certain metals are known for their toughness, thermal conductivity and good working characteristics and these can be advantageously combined with ceramic fibres known for their stiffness, strength and low temperature coefficient. In the case of synthetic materials, properties such as plasticity, hardness, elasticity, fracture strength and thermal and chemical resistivity can actually be "selected" by a suitable choice of starting materials, manufacturing process and additives.

\section{Joining as a challenge}

The idea of combining several materials to create a single part, with the aim of accumulating and enhancing the positive characteristics of the individual components, is a seductive one. In practice, however, materials cannot simply be combined at will. Their properties are often too different. Joining is also often a challenge. Whereas, for example, with a "classical" material such as steel several individual components can be welded together, this is not possible with ceramics and polymers. For that reason, other joining and connection techniques such as soldering or the use of adhesives must be considered.

\section{Electromagnetic actuator made of different materials}

This is the point at which the new EU research project takes over. The aim over the next three years is to develop innovative production and joining techniques for aerospace components. The sum of 4 million euros is available as part of the 7th EU Framework Program project.
The following companies and institutes are participating in the research project:

- Politecnico di Torino, Italy (Project Leader)

- MT Aerospace, Germany

- EADS, Germany

- Fraunhofer Gesellschaft, Germany

- Empa, Switzerland

- Consiglio Nationale delle Ricerche (CNR), Italy

- Nanoforce Technology, United Kingdom

Actively involved in the project are the High-Performance Ceramics Laboratory and the Joining Technologies and Corrosion Laboratory of Empa (Eidgenössischer Materialprüfungs- und Forschungsanstalt).The aim is to develop and manufacture an electromagnetic actuator of the kind used in aircraft or space vehicles to position aerodynamic control surfaces or for similar applications.

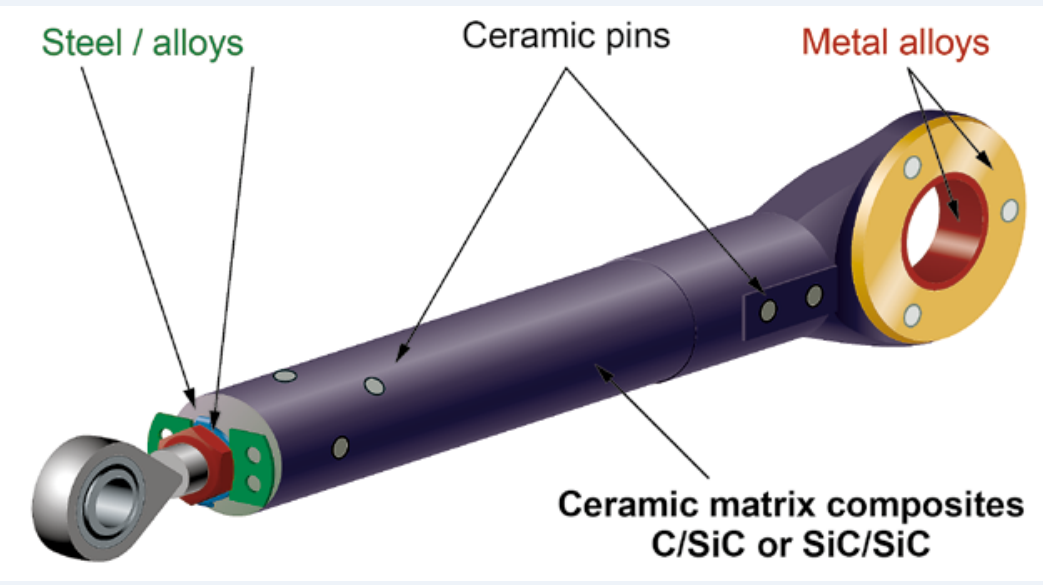

An electromagnetic actuator made of a combination of different materials: creating a functioning device from this combination of materials is the challenge facing researchers in the EU's "ADMACOM" project 